\title{
Nitric oxide-loaded echogenic liposomes for treatment of vasospasm following subarachnoid hemorrhage
}

This article was published in the following Dove Press journal:

International Journal of Nanomedicine

21 December 2013

Number of times this article has been viewed

\author{
Hyunggun Kim' \\ George L Britton' \\ Tao Peng' \\ Christy K Holland ${ }^{2}$ \\ David D McPherson' \\ Shao-Ling Huang' \\ 'Division of Cardiovascular Medicine, \\ Department of Internal Medicine, \\ The University of Texas Health \\ Science Center at Houston, \\ Houston, TX, USA; ${ }^{2}$ Division of \\ Cardiovascular Diseases, Department \\ of Internal Medicine, and Department \\ of Biomedical Engineering and \\ Radiology, University of Cincinnati, \\ Cincinnati, OH, USA
}

\begin{abstract}
Delayed cerebral vasospasm following subarachnoid hemorrhage causes severe ischemic neurologic deficits leading to permanent neurologic dysfunction or death. Reduced intravascular and perivascular nitric oxide (NO) availability is a primary pathophysiology of cerebral vasospasm. In this study, we evaluated NO-loaded echogenic liposomes (NO-ELIP) for ultrasound-facilitated NO delivery to produce vasodilation for treatment of vasospasm following subarachnoid hemorrhage. We investigated the vasodilative effects of $\mathrm{NO}$ released from NO-ELIP both ex vivo and in vivo. Liposomes containing phospholipids and cholesterol were prepared, and NO was encapsulated. The encapsulation and release of NO from NO-ELIP were determined by the syringe/vacuum method and ultrasound imaging. The ex vivo vasodilative effect of NO-ELIP was investigated using rabbit carotid arteries. Arterial vasodilation was clearly observed with NO-ELIP exposed to Doppler ultrasound whereas there was little vasodilative effect without exposure to Doppler ultrasound in the presence of red blood cells. Penetration of NO into the arterial wall was determined by fluorescent microscopy. The vasodilative effects of intravenously administered NO-ELIP in vivo were determined in a rat subarachnoid hemorrhage model. NO-ELIP with ultrasound activation over the carotid artery demonstrated effective arterial vasodilation in vivo resulting in improved neurologic function. This novel methodology for ultrasound-controlled delivery of NO has the potential for therapeutic treatment of vasospasm following subarachnoid hemorrhage. This ultrasound-controlled release strategy provides a new avenue for targeted bioactive gas and therapeutic delivery for improved stroke treatment.
\end{abstract}

Keywords: vasospasm, vasodilation, subarachnoid hemorrhage, nitric oxide, liposomes, ultrasound

\section{Introduction}

A number of bioactive gases such as nitric oxide (NO), ${ }^{1}$ hydrogen sulfide, ${ }^{2,3}$ carbon monoxide, ${ }^{4-6}$ and xenon ${ }^{7}$ have potent therapeutic effects. A typical method for delivery of therapeutic gases is inhalation. The US Food and Drug Administration approved inhaled NO for treatment of patients with persistent pulmonary hypertension. ${ }^{8}$ However, efficient delivery of a sufficient amount of NO to target tissues by inhalation is challenging due to the presence of endogenous NO scavengers such as hemoglobin in the circulation. ${ }^{9}$ Therefore, a more effective delivery strategy is required for local delivery of therapeutic gases.

NO plays a primary role in the regulation of vascular tone. ${ }^{10}$ Cerebral vasospasm following subarachnoid hemorrhage causes severe ischemic neurologic deficits leading to permanent neurologic dysfunction or death. ${ }^{11}$ Reduced availability of NO in blood and cerebrospinal fluid is one of the important mechanisms underlying cerebral
Correspondence: Shao-Ling Huang Division of Cardiovascular Medicine, The University of Texas Health Science Center at Houston, 643I Fannin St, MSB I.246, Houston, TX 77030, USA $\mathrm{Tel}+\mathrm{I} 7134862304$

Fax +I 7/35006556

Email shaoling.huang@uth.tmc.edu 
vasospasm, a complication of subarachnoid hemorrhage. ${ }^{11-13}$ Hemoglobin released following aneurysmal rupture inhibits NO production of endothelial nitric oxide synthase and decreases NO availability for smooth muscle cells, leading to vasoconstriction. ${ }^{11-13}$ Successful delivery of NO to the cerebral arteries may help prevent the genesis of cerebral vasospasm and provide cerebral vascular relaxation in patients with subarachnoid hemorrhage. ${ }^{14}$

Ultrasound contrast agents usually contain a perfluorcarbon gas stabilized by a shell of biocompatible material such as protein, ${ }^{15}$ lipid, ${ }^{16-19}$ or polymer. ${ }^{20}$ We have recently developed a novel methodology to encapsulate bioactive gases replacing air into our liposomal nanovehicles. ${ }^{21-25}$ Coencapsulation of NO with argon enabled us to adjust the amount of encapsulated NO. A total of $10 \mu \mathrm{L}$ of gas was encapsulated into $1 \mathrm{mg}$ of liposomes. The release profile of NO from NO-loaded echogenic liposomes (NO-ELIP) demonstrated an initial rapid release followed by a slower release up to 8 hours. Sixty-eight percent of the vascular smooth muscle cells remained viable when incubated with $80 \mu \mathrm{g} / \mathrm{mL}$ of NO/argon (1/9)-ELIP for 4 hours. NO delivery to vascular smooth muscle cells by the use of NO/argon-ELIP was 7-fold greater than unencapsulated NO. We have found that NO/argon at a ratio of 1:9 in ELIP is an effective agent for delivering NO to vascular smooth muscle cells even in the presence of hemoglobin, an NO scavenger. We have demonstrated that both pulsed diagnostic ultrasound and continuous ultrasound can be utilized to enhance therapeutic release from ELIP. ${ }^{25-27}$

In this study, we evaluated NO-ELIP for ultrasoundfacilitated site-specific NO delivery to produce vasodilation affecting vasospasm following subarachnoid hemorrhage. We investigated the vasodilative effects of NO released from NO-ELIP following ultrasound exposure in in vitro, ex vivo, and in vivo models.

\section{Materials and methods Preparation of NO-ELIP}

For the in vitro and ex vivo studies, liposomes were composed of 1,2-dipalmitoyl-sn-glycero-3-ethylphosphocholine (Genzyme Corporation, Cambridge, MA, USA), 1,2-dioleoylsn-glycero-3-phosphocholine (DOPC, Avanti Polar Lipids, Alabaster, AL, USA), and cholesterol (Avanti Polar Lipids) at a molar ratio of 60:30:10.

For the in vivo study, liposomes were composed of 1,2dipalmitoyl-sn-glycero-3-phosphocholine (Avanti Polar Lipids), 1,2-dioleoyl-sn-glycero-3-phosphocholine, and cholesterol at a molar ratio of 60:30:10 with the addition of $6 \%$ (molar ratio) carbonyl-methoxypolyethyleneglycol2000-2-dipalmitoyl-sn-glycero-3-phosphoethanolamine (PEG2000-DPPE) for prolonged circulating time. Addition of PEG-phosphoethanolamine improves circulation time. In a previous study, we have shown intravenous injection of this ELIP formulation with ultrasound release over the carotid artery resulted in an enhanced effect using another bioactive gas encapsulated ELIP. ${ }^{26}$

Lipids $(5 \mathrm{mg}$ ) were mixed in chloroform and vaporized with argon in $50^{\circ} \mathrm{C}$ water bath to form a thin film on a glass vial wall. The lipid film was placed under high vacuum $(<100$ Torr) for 4-6 hours to remove the solvent completely. The dried lipid film was hydrated with $0.32 \mathrm{M}$ deoxygenated mannitol and sonicated for 5 minutes. The sonicated liposomes were transferred to a $2 \mathrm{~mL}$ glass vial capped with a Teflon rubber septum. A mixture $(10 \mathrm{~mL})$ of NO (Specialty Gases of America Inc, Toledo, OH, USA) and argon (Matheson Tri-Gas, Houston, TX, USA) at a ratio of 1:9 was purified by bubbling through deoxygenated $5 \mathrm{M} \mathrm{NaOH} .{ }^{25}$ The gaseous mixture of NO and argon was injected into the glass vial through a Teflon rubber septum using a $12 \mathrm{~mL}$ syringe attached to a $27 \mathrm{G} \times 0.5$ inch needle. The pressurized liposomal dispersion was frozen at $-70^{\circ} \mathrm{C}$ for 30 minutes.

In order to quantitate the amount of $\mathrm{NO}$ encapsulated in NO-ELIP, the vial cap was removed, the pressure in the vial released, and the liposomal dispersion allowed to thaw. Passive release profiles of NO gas from the NO-ELIP were determined in vitro by measuring the remaining amount of gas in the NO-ELIP at room temperature under atmospheric pressure.

\section{In vitro ultrasound-triggered $\mathrm{NO}$ release from NO-ELIP}

Encapsulation and release of NO from NO-ELIP was determined using a previously developed syringe/vacuum method, ${ }^{19}$ and confirmed by measuring the echogenicity of the liposomes using transvascular ultrasound imaging. NO-ELIP $(380 \mu \mathrm{L})$ was added into $19 \mathrm{~mL}$ of phosphate-buffered saline (PBS) (Gibco ${ }^{\mathrm{TM}}$, Invitrogen $\mathrm{Co}$, Carlsbad, CA, USA) to obtain a $50 \times$ dilution factor. Diluted NO-ELIP $(4 \mathrm{~mL})$ was injected into a $5 \mathrm{~cm}$ long latex tubing (1/4 inch ID, 3/8 inch OD, 1/16 inch TH, McMaster-Carr, Atlanta, GA, USA) submerged in degassed water above an acoustic absorber pad (Precision Acoustics LTD, Dorchester, Dorset, UK) at room temperature under atmospheric pressure. A transvascular ultrasound transducer (Vivid i, GE Healthcare, Milwaukee, WI, USA) was placed $5 \mathrm{~mm}$ above the tubing, and $5.7 \mathrm{MHz}$ color Doppler ultrasound (mechanical index 0.15, pulse 
repetition frequency $8 \mathrm{kHz}$ ) was applied for 60 seconds for ultrasound-triggered gas release. The amount of remaining gas in the NO-ELIP was measured at 0, 10, 20, 30, 40, and 60 seconds after exposure to Doppler ultrasound. The amount of NO released $\left(\mathrm{V}_{\text {released }}\right)$ from the NO-ELIP was determined by subtracting the remaining $\mathrm{NO}$ amount $\left(\mathrm{V}_{\text {post }}\right)$ from the initial NO amount $\left(\mathrm{V}_{\text {pre }}\right.$ at time 0$)$ in the NO-ELIP at each time point. Ultrasound-triggered NO release data at 60 seconds was compared with the passive release (ie, without exposure to Doppler ultrasound). The enhanced NO release by Doppler ultrasound activation was confirmed by measuring the change of NO-ELIP echogenicity in B-mode ultrasound image data collected while applying Doppler ultrasound. Echogenicity of NO-ELIP inside the latex tubing $(20 \mathrm{~mm} \times 3 \mathrm{~mm}$ ) was determined using Image-Pro Plus software (Media Cybernetics Inc, Bethesda, MD, USA).

\section{Ex vivo ultrasound-controlled NO-ELIP delivery for vasodilation}

All animal experiments were approved by the animal welfare committee at The University of Texas Health Science Center at Houston. New Zealand White rabbits $(n=5)$ weighing $3.0-4.5 \mathrm{~kg}$ were anesthetized with ketamine $35 \mathrm{mg} / \mathrm{kg}$, xylazine $5 \mathrm{mg} / \mathrm{kg}$, and $1 \%-3 \%$ isoflurane. Rabbit erythrocytes were obtained prior to sacrifice of the animals and kept at $0^{\circ} \mathrm{C}$. Erythrocytes were diluted to $10 \%$ using PBS and the diluted red blood cell solution was utilized for the ex vivo experiments. Carotid arteries were harvested after sacrifice. The arteries were kept fresh in PBS at $-4^{\circ} \mathrm{C}$ and cut into 15 segments within 2 hours following excision.

Due to the ultrasound reflectivity in the currently available perfusion myograph systems, we designed a water bath with an acoustic absorber on the bottom to minimize ultrasound reflection. A one-way flow system was utilized to allow the rabbit carotid arteries to be perfused and exposed to $5.7 \mathrm{MHz}$ color Doppler ultrasound (mechanical index 0.15 , pulse repetition frequency $8 \mathrm{kHz}$ ) at room temperature (Figure 1A). A $500 \mathrm{~mL}$ stock reservoir of PBS with or without $10 \%$ of rabbit erythrocytes was placed $106.68 \mathrm{~cm}$ above the level where the artery was located to create pressure on the artery mimicking physiologic blood pressure. Latex tubing was used to connect the reservoir to the artery. The artery was immersed in the flow chamber filled with PBS. An acoustic absorber was placed beneath the artery to minimize ultrasonic reflection from the tank bottom. A transvascular ultrasound transducer (Vivid i) was placed $0.5 \mathrm{~cm}$ above the artery for ultrasound exposure. B-mode ultrasound imaging was used to measure changes in the vessel diameter and color Doppler mode was used to trigger the release of NO from NO-ELIP.

Four treatment groups were tested: NO-ELIP treatment without ultrasound exposure in the absence of erythrocytes, NO-ELIP treatment with ultrasound exposure in the absence of erythrocytes, NO-ELIP treatment without ultrasound exposure in the presence of erythrocytes, and NO-ELIP treatment with ultrasound exposure in the presence of erythrocytes. B-mode ultrasound imaging of the arterial segment was performed prior to NO-ELIP injection in the flow system at baseline. Using the same transvascular ultrasound transducer, color Doppler mode was activated over the arterial segment for 30 seconds for the ultrasound exposure groups. B-mode ultrasound images were recorded again 2 minutes following NO-ELIP injection to measure changes in arterial diameter.

Ultrasound images of the arterial segment along the axial direction were recorded in digital format using the transvascular ultrasound system, and transferred to a personal computer to quantitate the arterial diameters. Figure 1B demonstrates a representative B-mode ultrasound image of a rabbit arterial segment. Both edges of the near-wall and far-wall of the artery can be clearly distinguished. The luminal diameter of each artery before and 2 minutes following NO-ELIP injection was measured using Image-Pro Plus software. The arterial diameter was measured at three locations (Figure 1B) across the arterial segment and averaged.

Specific uptake of NO by vascular smooth muscle cells in the arterial wall was determined by the 4,5-diaminofluorescein diacetate (DAF-2DA) fluorometric detection system (Calbiochem, San Diego, CA, USA). DAF-2DA is a nonfluorescent agent that can readily diffuse into cells and be converted into DAF-2 by cytosolic esterase inside living cells. ${ }^{28}$ When NO is delivered into vascular smooth muscle cells, NO reacts with DAF-2, a non-fluorescent dye, to generate DAF-2T, a fluorescent triazole derivative.

Following ultrasound image data acquisition, the arteries were sliced at $4 \mathrm{~mm}$ intervals, immersed in optimal cutting temperature compound, frozen on dry ice, and stored at $-80^{\circ} \mathrm{C}$. Frozen sections from the NO-ELIP treatment groups were cut into $10 \mu \mathrm{m}$ thickness using a cryostat and mounted on glass slides. DAF-2DA working solution (10 $\mu \mathrm{L}$, 1:500 diluted in PBS) was added to each slide as a NOfluorescent agent. ${ }^{29}$ The uptake of NO by vascular smooth muscle cells was assessed by fluorescence microscopy at $40 \times$ and $100 \times$ magnifications. Distribution and penetration of NO across the arterial wall with or without Doppler ultrasound exposure were determined. 

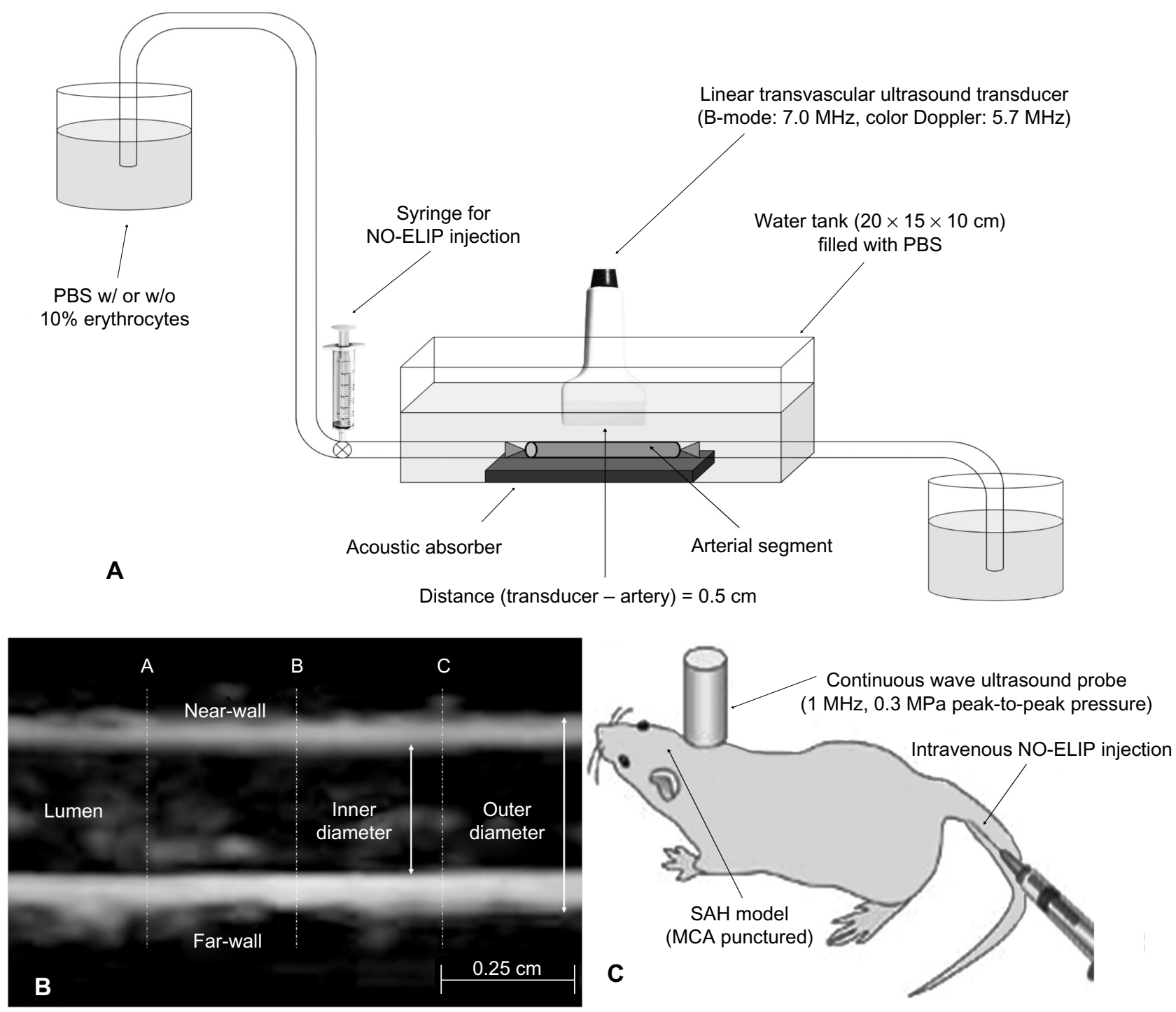

Figure I (A) Schematic of the ex vivo experimental setup with a rabbit carotid artery to investigate the vasodilative effects of NO-ELIP with and without ultrasound application. (B) Representative B-mode ultrasound image of a rabbit carotid arterial segment. Both edges of the near wall and far wall of the artery can be clearly distinguished. (C) Schematic of the in vivo experimental setup to determine vasodilative effects of ultrasound-controlled NO-ELIP delivery. The ultrasound probe was placed over the carotid artery to trigger NO release from the intravenously administered NO-ELIP.

Abbreviations: NO, nitric oxide; NO-ELIP, NO-loaded echogenic liposomes; PBS, phosphate-buffered saline; w/, with; w/o, without; SAH, subarachnoid hemorrhage; MCA, middle cerebral artery.

\section{In vivo ultrasound-controlled NO-ELIP delivery for cerebral vasodilation}

A total of 20 Sprague Dawley male rats (260-280 g; Harlan Laboratories Inc., Indianapolis, IN, USA) were utilized to determine the vasodilative effect of NO delivered by NO-ELIP following subarachnoid hemorrhage in vivo (Figure 1C). Anesthesia was induced by intraperitoneal injection of a cocktail solution of ketamine $25 \mathrm{mg} / \mathrm{mL}$, diazepam $2 \mathrm{mg} / \mathrm{mL}$, and atropine $0.1 \mathrm{mg} / \mathrm{mL}$ at a dose of $2.5 \mathrm{~mL} / \mathrm{kg}$ prior to surgery. Bupivacaine $0.25 \%$ (Hospira, Inc, Lake Forest, IL, USA) $2.0 \mathrm{mg} / \mathrm{kg}$ was injected subcutaneously to provide topical analgesia at the surgical site. During the operation, a temperature-controlled heating pad was used to maintain the rectal temperature at $37.5^{\circ} \mathrm{C}$.
Six groups were evaluated, comprising sham control, subarachnoid hemorrhage control, NO-ELIP treatment following subarachnoid hemorrhage, NO-ELIP with ultrasound exposure following subarachnoid hemorrhage, exposure to ELIP without NO loading following subarachnoid hemorrhage, and NO solution following subarachnoid hemorrhage. The internal carotid artery was exposed through a midline neck incision using an operating microscope. Subarachnoid hemorrhage was induced by endovascular perforation of the right middle cerebral artery using a sharp tip 3-0 nylon monofilament. ${ }^{30}$ Regional blood flow in the middle cerebral artery was monitored during the surgery using a laser Doppler flow meter to ensure the proper puncture 
on the artery. An immediate decrease in cerebral blood flow in the middle cerebral artery supplying area was considered as a sign of subarachnoid hemorrhage induction. Rats without a cerebral blood flow decrease were excluded.

For the treatment groups, NO-ELIP $(500 \mu \mathrm{g}, 1 \mathrm{mg} / \mathrm{mL})$ was infused via the right femoral vein at 30 minutes after subarachnoid hemorrhage onset for a period of 45 minutes. For the ultrasound exposure group, a $1 \mathrm{MHz}$ ultrasound probe (Sonitron 2000, Rich-Mar Corporation, Inola, OK, USA) was placed over the internal carotid artery to trigger NO release from NO-ELIP during NO-ELIP infusion. Ultrasound gel was used to fill the interface between the internal carotid artery and the ultrasound probe to ensure adequate acoustic coupling. Continuous wave ultrasound was set with a peak-to-peak pressure amplitude of $0.30 \mathrm{MPa}\left(1 \mathrm{~W} / \mathrm{cm}^{2}\right.$, $50 \%$ duty cycle). ${ }^{31}$

Neurologic assessment was performed 24 hours after subarachnoid hemorrhage using three different behavioral tests. ${ }^{26,32}$ All behavioral tests were conducted in a quiet and low-lit room by an observer blinded with respect to the treatment groups. Each animal was tested for motor function and neurologic outcomes by recording their ability to perform limb placing, beam walking, and grid walking on the day following subarachnoid hemorrhage. ${ }^{26}$

Following the behavioral tests, a second dose of NOELIP (confirmation of vasodilation) was administered for 10 minutes via the tail vein. Ultrasound was again used for additive NO release in group 4 (NO-ELIP with ultrasound exposure). Since we can only histologically measure the luminal dimension after NO-ELIP injection, in order to observe the immediate vasodilative effect of $\mathrm{NO}$ delivered by NO-ELIP, the rat was perfused with $300 \mathrm{~mL}$ of PBS solution and reperfused with a mixture of $4 \%$ paraformaldehyde and $2.5 \%$ glutaraldehyde by means of transthoracic cannulation of the left ventricle immediately after NO-ELIP injection. The brains were immediately harvested and placed in the fixative solution for 24 hours. Coronal sections were cut every $500 \mu \mathrm{m}$ over the area affected by subarachnoid hemorrhage ( 15 sections per animal) and stained with hematoxylin and eosin. The degree of vasospasm was determined by measuring luminal areas of the posterior communicating artery. The ratio of luminal area to total arterial area was calculated by image analysis.

\section{Statistical analysis}

SigmaStat (version 3.5, Systat Software Inc, Point Richmond, CA, USA) was utilized for data analysis. The Wilcoxon rank sum test was utilized for comparison of two groups.
For comparisons of multiple groups, the Kruskal-Wallis analysis of variance of ranks and median test were used to assess differences between the treatment groups. A value of $P<0.05$ was considered to be statistically significant. All data are presented as the mean with the standard error.

\section{Results}

\section{Encapsulation and release of $\mathrm{NO}$}

Encapsulation and release of NO from NO-ELIP was determined. The average volume of encapsulated $\mathrm{NO} /$ argon mixture in the NO-ELIP was $45 \mu \mathrm{L} \pm 13 \mu \mathrm{L}$. This volume remained stable ( $41 \mu \mathrm{L} \pm 15 \mu \mathrm{L})$ at room temperature under atmospheric pressure over 60 seconds without exposure to ultrasound, ie, under passive release (Figure 2A). Upon exposure to Doppler ultrasound, $21 \%$ and $50 \%$ of the encapsulated gas was released at 10 and 30 seconds, respectively. Release of the entrapped gas was almost complete at 60 seconds under exposure to Doppler ultrasound.

The mean grayscale values ( 0 , black; 255, white) of the region of interest filled with NO-ELIP inside the latex tubing were measured (Figure 2B and C). The initial mean grayscale value prior to Doppler ultrasound application was $106 \pm 33$. This decreased by approximately $55 \%$ at 30 seconds under exposure to Doppler ultrasound, and reached $25 \pm 10$ (76\% decrease) at 60 seconds. The decreased echogenicity over time corresponded to the NO release profile from NO-ELIP (Figure 2A).

\section{Ex vivo vasodilation by ultrasound- controlled NO delivery}

Vasodilative effects caused by NO-ELIP treatment in the rabbit carotid artery were investigated in the ex vivo model with respect to ultrasound exposure in the presence of red blood cells (Figure 3). In the absence of erythrocytes (ie, only PBS), NO-ELIP exposed to Doppler ultrasound induced greater arterial dilation $(6.6 \% \pm 3.6 \%)$ than without exposure to Doppler ultrasound $(2.9 \% \pm 2.6 \%)$. In the presence of erythrocytes, NO-ELIP demonstrated vasodilative effects only with Doppler ultrasound activation $(2.9 \% \pm 1.8 \%)$. There was no vasodilation without Doppler ultrasound, presumably due to the scavenging effect of the erythrocytes.

Penetration of NO released from NO-ELIP into the arterial wall is demonstrated in Figure 4. Distribution of NO across the arterial wall is colored green using DAF-2DA fluorometry. Without exposure to Doppler ultrasound, the media appeared pale green presumably due to interference from the cytosol of the arterial cells, and NO penetration was displayed primarily in the vicinity of the intima. Deeper and 
A

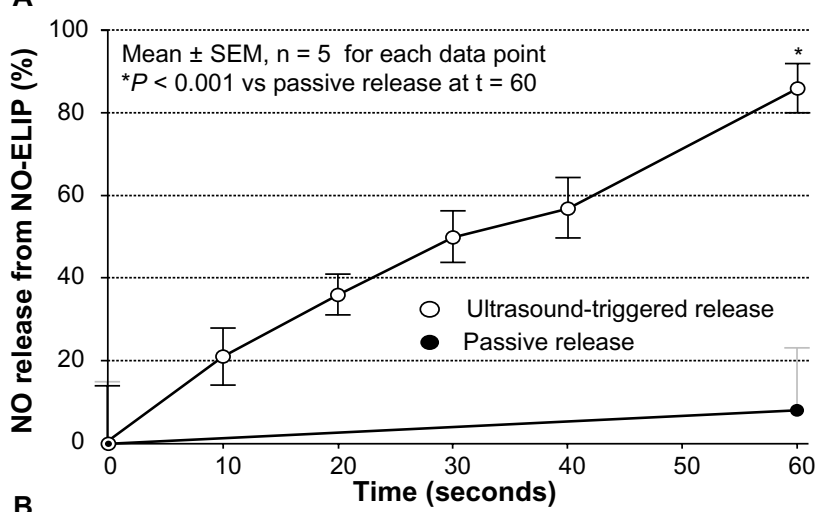

B

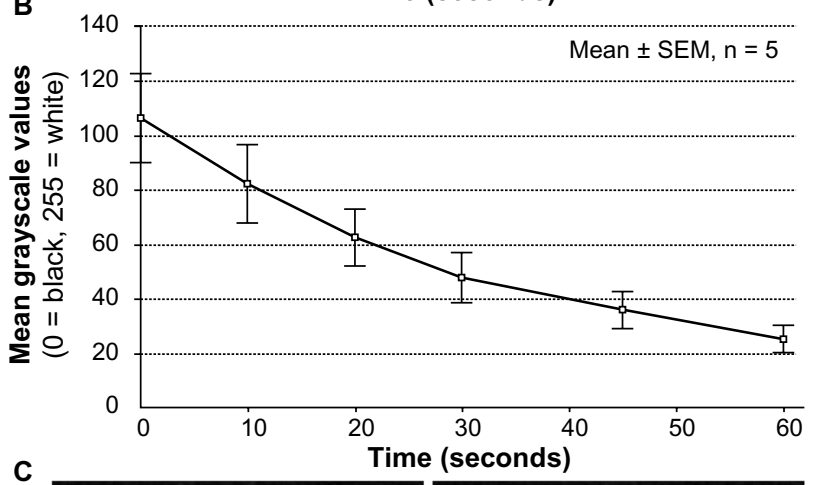

C

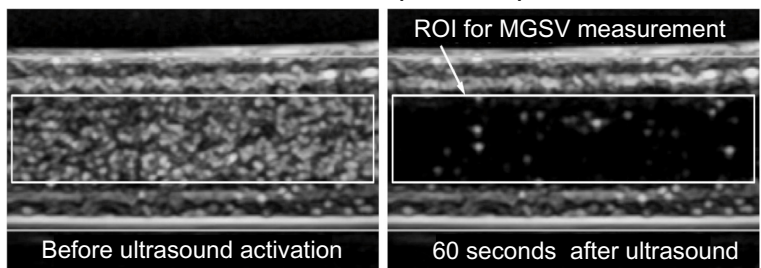

Figure 2 (A) Percentage changes of the released gas amount from NO-ELIP with and without Doppler ultrasound application. (B) Changes in echogenicity of NOELIP under Doppler ultrasound exposure. Echogenicity of NO-ELIP decreased as the encapsulated NO was released. (C) Representative B-mode ultrasound images of NO-ELIP under flow inside the latex tubing before and 60 seconds after ultrasound activation demonstrating the change of echogenicity.

Abbreviations: NO, nitric oxide; NO-ELIP, NO-loaded echogenic liposomes; SEM, standard error of the mean; vs, versus; ROI, region of interest; MGSV, mean grayscale value.

greater penetration of NO into the intima, media, and adventitia was clearly observed when NO-ELIP were exposed to Doppler ultrasound. The nuclei of the cells appear blue in the fluorescent images.

\section{In vivo ultrasound-controlled NO-ELIP delivery for cerebral vasodilation}

The in vivo vasodilative effect of intravenously administered NO-ELIP in combination with ultrasound application was determined by histologic analysis and behavioral tests. The ratio of luminal area to total arterial area in the sham model was $0.48 \pm 0.02$ (Figure 5). Following subarachnoid hemorrhage, the ratio of luminal area to total arterial area decreased to $0.35 \pm 0.04$ ( $P<0.05$ versus sham). NO-ELIP inhibited subarachnoid hemorrhage-induced vasospasm and the average ratio of luminal area to total arterial area was $0.46 \pm 0.04$ ( $P<0.05$ versus subarachnoid hemorrhage). Both ELIP only and NO solution treatments revealed little effect on the ratio of luminal area to total arterial area $(0.33 \pm 0.03$ and $0.34 \pm 0.03$, respectively). Although ultrasound activation with NO-ELIP administration further increased the inhibition of vasospasm resulting in the ratio of luminal area to total arterial area of $0.48 \pm 0.02(P<0.05$ versus subarachnoid hemorrhage, $P=0.74$ versus subarachnoid hemorrhage + NOELIP), there was no difference in the ratio of luminal area to total arterial area between the NO-ELIP and NO-ELIP/ ultrasound treatment groups. This may be due to giving the NO-ELIP or NO ELIP/US treatment on day 2 of subarachnoid hemorrhage (this experiment was used to confirm vasodilation; not for quantifying the degree of vasodilation).

Behavioral tests demonstrated improved neurologic function for both the NO-ELIP and NO-ELIP/US treatment groups (Figure 6). Animals without treatment following subarachnoid hemorrhage showed substantial deficits in locomotor performance. NO-ELIP treatment improved locomotor performance in all behavioral tests. Animals with NO-ELIP treatment combined with ultrasound exposure demonstrated further improved neurologic functions overall in all behavioral tests.

\section{Discussion}

The present study demonstrates the feasibility of NO-loaded liposomes for acute vasodilation. The use of ELIP as a gas delivery vehicle can protect NO gas from scavenging by circulating hemoglobin. Ultrasound exposure has the potential to release the gaseous payload to target tissues for enhanced therapeutic effect. This strategy can provide efficient NO delivery to generate sufficient local NO concentration for vasodilative and neuroprotective effects.

In addition to protecting NO in the circulation and facilitating ultrasound-controlled release, ELIP have many other advantages as bioactive gas delivery agents. First, the gas concentration encapsulated in ELIP can be adjusted by changing the ratio of $\mathrm{NO}$ and argon gases or by changing the magnitude of pressure in the preparation procedure. In this study, a mixture of $\mathrm{NO}$ and argon at a ratio of 1:9 encapsulated under 9 atm pressure was utilized to produce a total volume of $45 \mu \mathrm{L} \pm 13 \mu \mathrm{L}$ encapsulated into $5 \mathrm{mg}$ of lipids. ${ }^{33}$ Second, the average size of our ELIP is $800 \mathrm{~nm}$. They can be conjugated with antibodies for targeted delivery. ${ }^{22,34}$ Other investigators have employed similar strategies using immunoliposomes targeted to inflammation in myocardial infarction. Third, ELIP can have both hydrophobic and hydrophilic cores due to its 


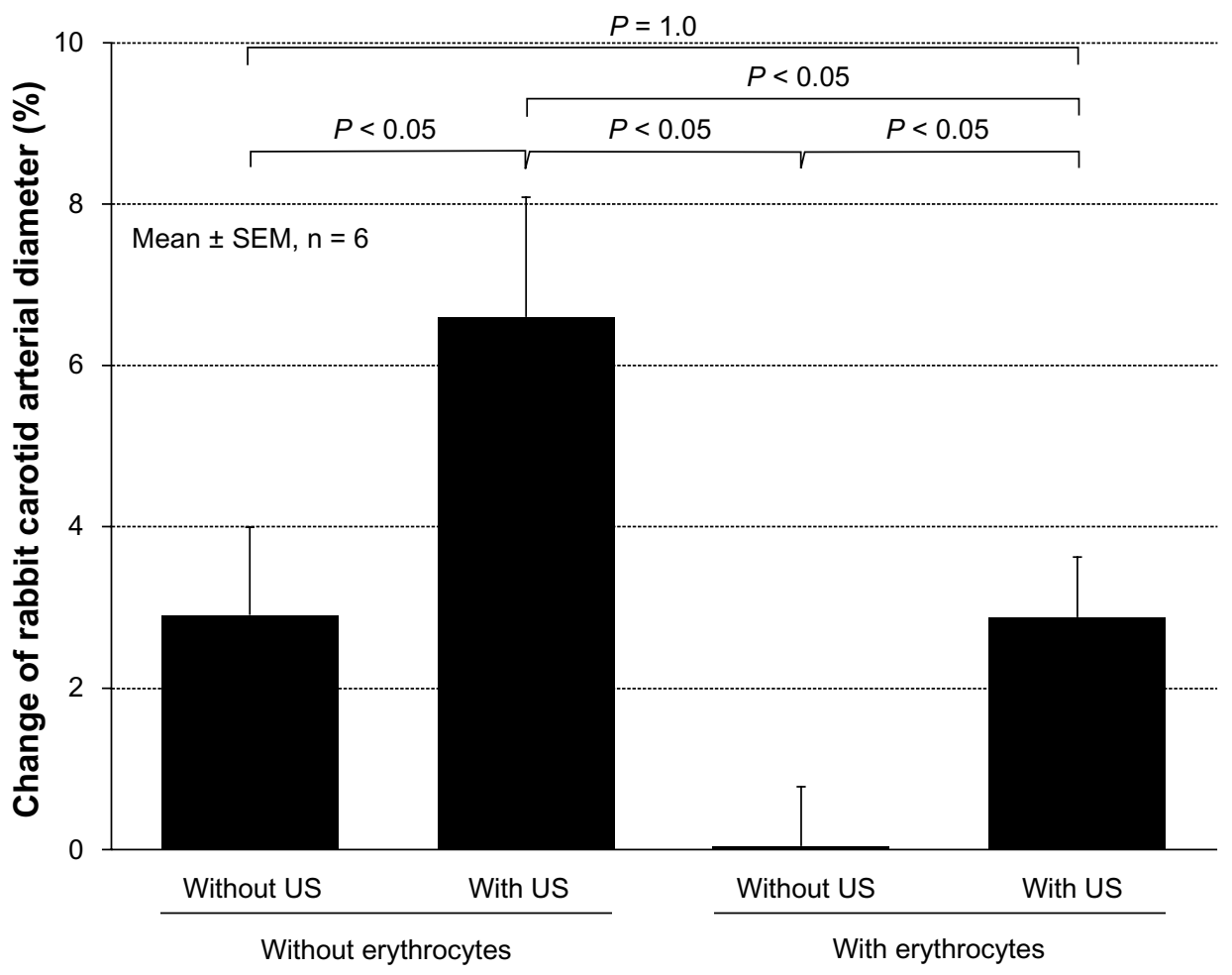

Figure 3 Arterial vasodilation induced by NO-ELIP with and without exposure to pulsed Doppler ultrasound in the absence and presence of erythrocytes. Abbreviations: NO, nitric oxide; NO-ELIP, NO-loaded echogenic liposomes; SEM, standard error of the mean; US, ultrasound.

bilayered lipid structure. Gas bodies are considered hydrophobic and are likely encapsulated in the liposomal hydrophobic core or within the lipid monolayer inside the liposomes. This provides gas-loaded liposomes with the capability of coencapsulating a hydrophilic drug such as sildenafil, a potent and selective inhibitor of cGMP-specific phosphodiesterase type 5, into the liposomal hydrophilic core, potentially enabling controlled release of both a gas and a drug from these ELIP.

Acoustic cavitation is the process of formation and collapse of gas bubbles in a liquid due to acoustic pressure. Cavitation can be divided into two types, ie, noninertial (or stable) cavitation and inertial (or transient) cavitation. Noninertial cavitation is defined as cavitation bubbles expanding and contracting upon acoustic rarefaction and compression, without growing to critical collapse size.

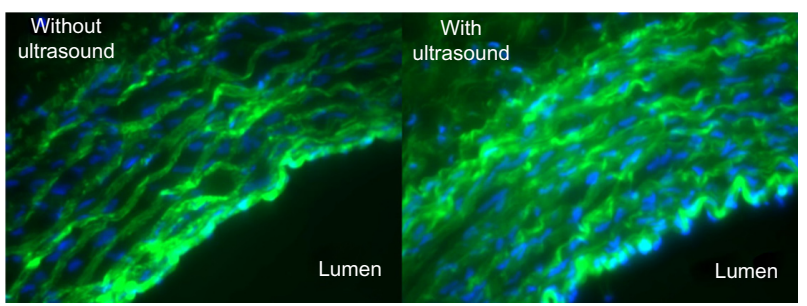

Figure 4 Penetration of NO released from NO-ELIP into a rabbit carotid arterial wall with and without pulsed Doppler ultrasound application in an ex vivo setting. Abbreviations: NO, nitric oxide; NO-ELIP, NO-loaded echogenic liposomes.
Inertial cavitation is characterized by the rapid collapse of microbubbles, leading to fragmentation. ${ }^{35}$

We examined two different types of ultrasound, pulsed Doppler and continuous (mechanical) ultrasound, to investigate the vasodilative effects of ultrasound-triggered $\mathrm{NO}$ release from NO-ELIP. Arterial vasodilation was clearly observed with NO-ELIP exposed to pulsed Doppler ex vivo compared to little vasodilative effect without exposure to Doppler in the presence of red blood cells (Figure 3). We have previously demonstrated that pulsed Doppler ultrasound with a center frequency of $6.0 \mathrm{MHz}$ and mechanical index value between 0.1 and 0.5 induces acoustically driven gas diffusion with air-containing ELIP. ${ }^{36}$ This finding has practical importance because low acoustic pressures without inertial cavitation can potentially produce less or no damage to biological tissues. NO-ELIP with continuous mechanical ultrasound activation over the carotid artery also demonstrated effective arterial vasodilation in vivo, resulting in improved neurologic function (Figure 5). Further studies will more accurately determine the relationship between the types of ultrasound, ultrasound parameters, and rate and amount of gas release under various blood flow rates for in vivo applications.

NO has numerous and diverse biological activities, but as a gas it is too labile for vascular delivery in vivo. The half-life of NO in the circulation is only 1.8 milliseconds. 


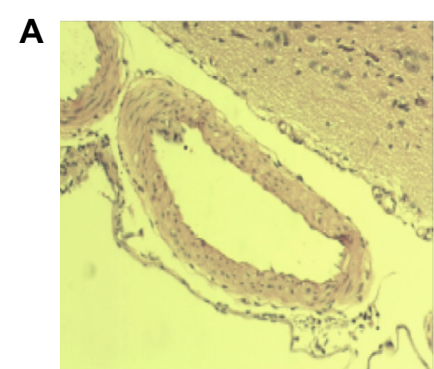

Sham

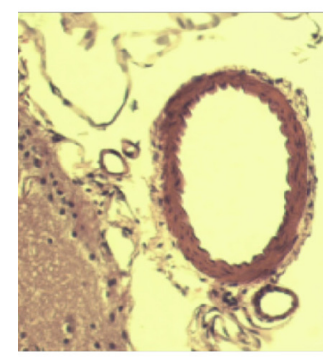

SAH + NO-ELIP

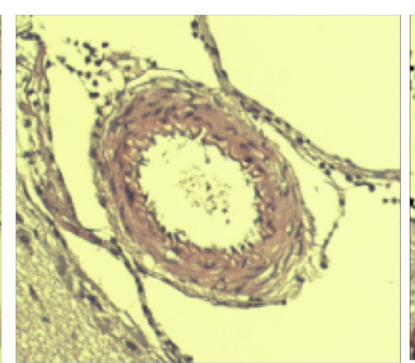

$\mathrm{SAH}$
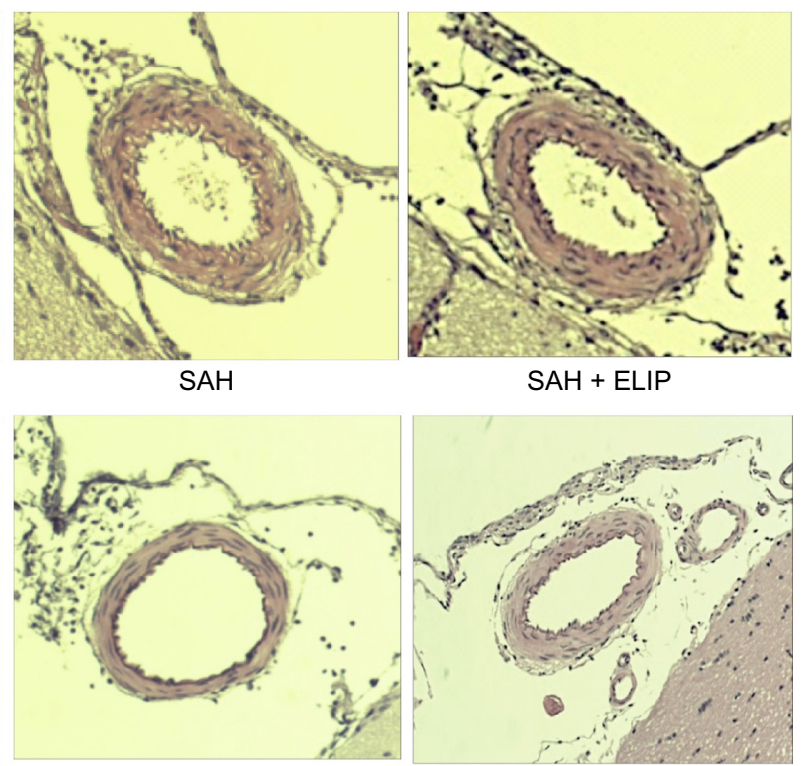

SAH + NO-ELIP/US

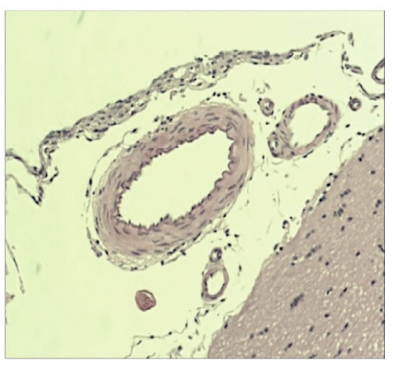

$\mathrm{SAH}+\mathrm{NO}-$ solution

в

$P=0.393$

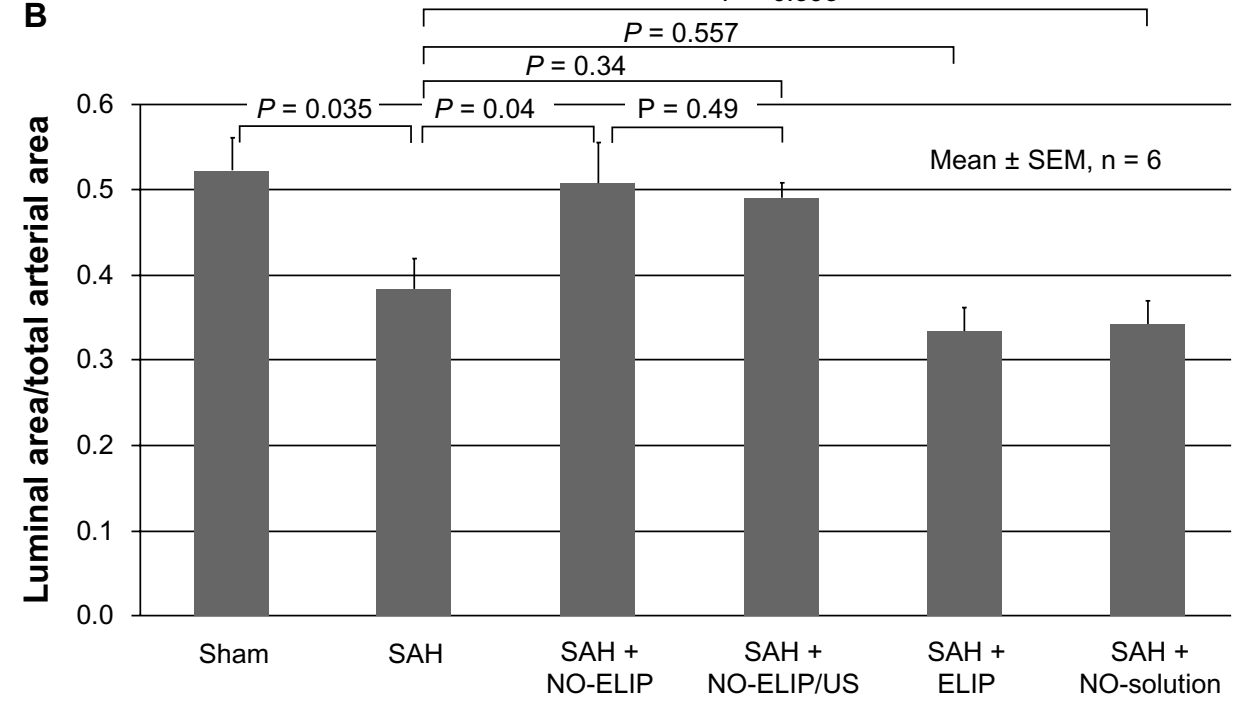

Figure 5 (A) Representative cerebral arterial segments stained with hematoxylin and eosin. (B) Normalized luminal area with respect to total arterial area demonstrating vasodilative effects of NO-ELIP on subarachnoid hemorrhage-induced vasospasm in a rat subarachnoid hemorrhage model.

Abbreviations: NO, nitric oxide; NO-ELIP, NO-loaded echogenic liposomes; SAH, subarachnoid hemorrhage; US, ultrasound; SEM, standard error of the mean.

A

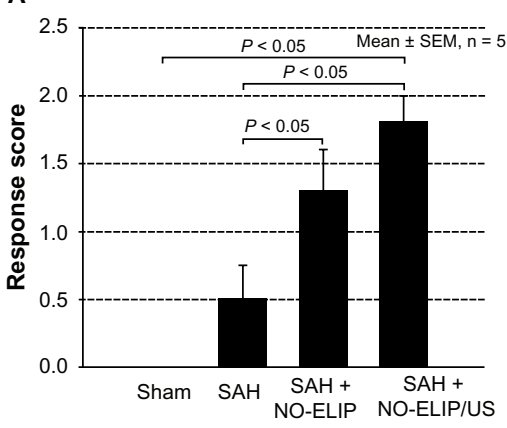

B

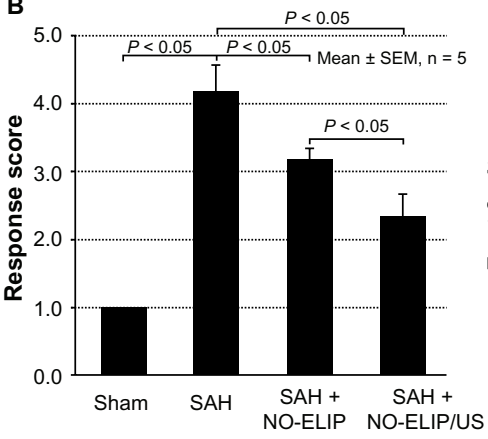

C

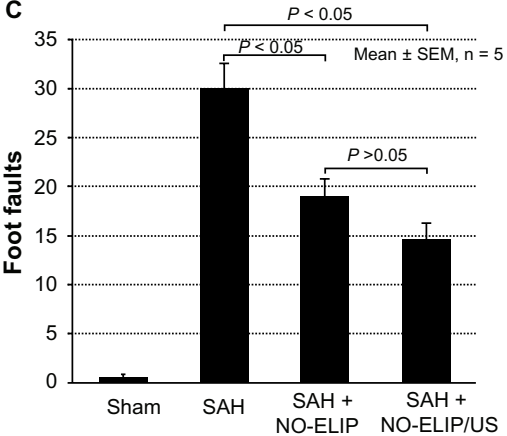

Figure 6 Behavioral assessment 24 hours after subarachnoid hemorrhage. (A) Limb placement, (B) beam walking, and (C) grid walking. All behavioral tests demonstrated improved neurologic function with NO-ELIP treatment in a rat subarachnoid hemorrhage model.

Abbreviations: NO, nitric oxide; NO-ELIP, NO-loaded echogenic liposomes; SEM, standard error of the mean; SAH, subarachnoid hemorrhage; US, ultrasound. 
Encapsulation of NO into ELIP can protect NO from scavenging by hemoglobin. To evaluate NO release from NO-ELIP, the encapsulation and release of NO from NOELIP were determined by measuring NO release in ELIP using a previously developed syringe/vacuum method. ${ }^{19}$ This method helps overcome drawbacks of chemical methods on detection/detectability of NO when NO scavengers are present. In order to evaluate the biologic effects of the NO released from NO-ELIP, vasodilation was studied in an open-circuit perfusion system with erythrocytes in perfusion solution. Our results demonstrated that encapsulation of NO into ELIP can protect NO from scavenging by hemoglobin.

Although encapsulation of NO into ELIP can protect NO from scavenging by erythrocytes, NO released locally from NO-ELIP can still be under the effect of erythrocytes if released too far from the arterial wall. This can be overcome by using positively charged NO-ELIP or antibody-conjugated NO-ELIP that can attach to the vascular endothelium prior to ultrasound application. In this study, we used positively charged liposomes that can attach to the vascular endothelium and successfully demonstrated vasodilation with ultrasound application. The development of site-specific targeted NOELIP will boost delivery efficiency. The bioactive gas delivery methodology described in this study can be utilized for protection and release of other therapeutic gases that have short survival time, lack of donors, or unpleasant inhalation odors.

Ultrasound not only triggers local NO release from NO-ELIP but also enhances NO penetration into the arterial wall. Although many therapeutic agents have demonstrated efficient pharmacologic effects in vascular diseases in vitro and with animal models, they often fail to achieve the same effects in humans. One of the most important contributing factors to this phenomenon is insufficient local penetration into the vascular wall. Even after reaching a sufficient local concentration, the transport of therapeutic agents from blood into the vascular wall through the endothelium often remains problematic. Uptake of therapeutic agents by the arterial wall is dependent on diffusion and transendothelial permeability as well as the molecular weight, hydrophobic/hydrophilic balance, and electrostatic charge of the therapeutic. ${ }^{37}$ Gases can be considered small molecules that can readily cross the endothelial barrier. ${ }^{38}$ A further advantage of ultrasound-controlled local release is the radiation force effects that can enhance the recruitment of liposomes into the arterial wall. ${ }^{39}$ Doppler ultrasound exposure for 60 seconds releases about $40 \mu \mathrm{L}$ of NO from
NO-ELIP, while less than $5 \mu \mathrm{L}$ of NO is released without triggering by ultrasound (Figure 2).

To visualize NO uptake into the arterial wall, DAF-2DA staining was performed on frozen tissue sections. A limitation of the DAF-2DA method for NO detection is autofluorescence from the artery. However, the most important goal using this approach was to visualize NO distribution. Our data demonstrated efficient NO diffusion into the intima but not in the media without exposure to ultrasound (Figure 4). With ultrasound activation, enhanced NO distribution was clearly observed across the entire arterial wall. This indicates that ultrasound effectively enhances the total amount of NO penetrating the arterial wall in an appropriately short time period. Histologic imaging confirmed close colocation of green fluorescence with cell nuclei (blue, diamidino2-phenylindole staining) in the media. This confirms NO distribution into the smooth muscle cells with resultant vasodilation. Further experiments using an autofluorescence quench method and precise quantitation of NO distribution in an arterial wall will further help us to understand the exact mechanisms of NO delivery.

In addition to being a potent regulator of cerebral arterial tone, NO can provide neuroprotection and antimicrothrombosis, increase cerebral ischemic tolerance, and promote endothelial cell function. ${ }^{40}$ Cerebral NO levels decrease in the first hours following subarachnoid hemorrhage and recover at 24 hours. ${ }^{41}$ In the present study, we delivered $\mathrm{NO}$ at 30 minutes after subarachnoid hemorrhage. It is clear that NO-ELIP improved neurologic function after subarachnoid hemorrhage. This occurred with or without ultrasound exposure. However, when ultrasound was applied, it further enhanced the effect of NO-ELIP on neurologic improvement without increasing the vasodilative effect (Figure 6). This indicates that neurologic improvement with ultrasound exposure can be related to both direct $\mathrm{NO}$ neuroprotective effects and indirect neuroprotection due to vasodilation. The second NO-ELIP injection at 24 hours after subarachnoid hemorrhage was meant to confirm NO-ELIPassociated vasodilation. It cannot answer the extra effect of ultrasound for greater NO release as evidenced in the neurologic behavior test results. We do not as yet know the optimal therapeutic dose of NO-ELIP. A higher concentration may be needed for maximal neuroprotection following subarachnoid hemorrhage. To confirm NO ELIP-induced vasodilation only, the second NO-ELIP administration was performed 24 hours after stroke onset.

Although our ultimate goal is to identify an optimal Doppler ultrasound pulse sequence for clinical gas or drug release 
from our ELIP, we used an ultrasound transducer specifically made for small animal use in our initial in vivo experiments. No matter which ultrasound instrument is ultimately used, the acoustic rarefactional pressure and center frequency (or mechanical index) are the key parameters. We have calibrated the acoustic output of the ultrasound transducer (Sonitron 2000) to determine accurately the pressure output and beam characteristics. ${ }^{31}$ An ultrasound probe $(8 \mathrm{~mm}$ diameter, $1.0 \mathrm{MHz}, 1 \mathrm{~W} / \mathrm{cm}^{2}, 50 \%$ duty cycle) with peak-to-peak acoustic pressure of $0.30 \mathrm{MPa}$ was used over the rat carotid artery to trigger NO release. For in vitro study, color Doppler ultrasound (5.7 MHz, mechanical index 0.15, pulse repetition frequency $8 \mathrm{kHz}$ ) with a similar acoustic rarefactional pressure was employed.

NO was used as a prototype therapeutic gas for ELIP entrapment. Our ELIP have the ability to encapsulate other bioactive gases, such as xenon, that can similarly increase endothelial and adventitial penetration upon release. This novel therapeutic gas delivery method provides a solid foundation for developing ultrasound-based strategies for targeted delivery of bioactive gases.

Precise in vivo evaluation of cerebral vasospasm caused by subarachnoid hemorrhage has remained an unsolved issue in experimental small animal models. In our studies, in vivo vasodilation was evaluated ex vivo by histologic methods. Features of modeling corrugated internal elastic lamina (Figure 5) and reduced lumen area indicated vasospasm. Although the endovascular perforation model mimics the events of human aneurysm rupture, future studies using a cranial window with an autologous blood injection approach may allow real-time observation of changes in blood vessel diameter. However, because the autologous blood injection approach does not exactly mimic human subarachnoid hemorrhage, other models may need to be developed to confirm NO-ELIP vasodilation further.

In the present study, ultrasound exposure demonstrated a further beneficial effect with improved neurologic function. This may be related to additive NO release from NO-ELIP. We do not as yet know the optimal dose of NO via NO-ELIP needed for sufficient penetration into neuronal cells for maximal neuroprotection following subarachnoid hemorrhage. Using a lower dose of NO-ELIP, ultrasound may have additive effects. This needs further investigation.

\section{Conclusion}

In this study, a novel methodology for ultrasound-controlled delivery of NO and the potential for therapeutic treatment of vasospasm following subarachnoid hemorrhage have been described. This technique provides desirable properties, including high payload concentration, modulatory gas release profiles, protection of bioactive gas payloads against exogenous scavengers, and successful payload delivery to vascular cells with biological effect. This novel ultrasoundcontrolled release strategy has the potential to open new avenues for targeted bioactive gas and therapeutic delivery for improved stroke treatment.

\section{Acknowledgments}

This work was supported in part by the National Institutes of Health (NS67454 to S-LH, HL74002 and HL59586 to DDM, and NS47603 to CKH). The authors thank Stephen Chrzanowski, Melanie Moody, Yuejiao Zhou, and Melvin Klegerman for their assistance.

\section{Disclosure}

HK, DDM, S-LH, and The University of Texas Health Science Center at Houston have research-related financial interests in Zymo Pharmaceuticals LLC (Irvine, CA, USA). The other authors have no conflicts of interest to declare for this work.

\section{References}

1. Furchgott RF, Zawadzki JV. The obligatory role of endothelial cells in the relaxation of arterial smooth muscle by acetylcholine. Nature. 1980;288(5789):373-376.

2. Martelli A, Testai L, Marino A, Breschi MC, Da Settimo F, Calderone V. Hydrogen sulphide: biopharmacological roles in the cardiovascular system and pharmaceutical perspectives. Curr Med Chem. 2012;19(20): 3325-3336.

3. Skovgaard N, Gouliaev A, Aalling M, Simonsen U. The role of endogenous H2S in cardiovascular physiology. Curr Pharm Biotechnol. 2011;12(9):1385-1393.

4. Choi YK, Por ED, Kwon YG, Kim YM. Regulation of ROS production and vascular function by carbon monoxide. Oxid Med Cell Longev. 2012;2012:794237.

5. Fujita K, Yamafuji M, Nakabeppu Y, Noda M. Therapeutic approach to neurodegenerative diseases by medical gases: focusing on redox signaling and related antioxidant enzymes. Oxid Med Cell Longev. 2012; 2012:324256.

6. Szabo C. Gaseotransmitters: new frontiers for translational science. Sci Transl Med. 2010;2(59):59ps4.

7. Dickinson R, Franks NP. Bench-to-bedside review: Molecular pharmacology and clinical use of inert gases in anesthesia and neuroprotection. Crit Care. 2010;14(4):229.

8. Griffiths MJ, Evans TW. Inhaled nitric oxide therapy in adults. $N$ Engl J Med. 2005;353(25):2683-2695.

9. Tsao PS, McEvoy LM, Drexler H, Butcher EC, Cooke JP. Enhanced endothelial adhesiveness in hypercholesterolemia is attenuated by L-arginine. Circulation. 1994;89(5):2176-2182.

10. Ignarro LJ. Nitric oxide as a unique signaling molecule in the vascular system: a historical overview. J Physiol Pharmacol. 2002;53(4 Pt 1): 503-514.

11. Pluta RM. Delayed cerebral vasospasm and nitric oxide: review, new hypothesis, and proposed treatment. Pharmacol Ther. 2005;105(1): 23-56. 
12. Pradilla G, Chaichana KL, Hoang S, Huang J, Tamargo RJ. Inflammation and cerebral vasospasm after subarachnoid hemorrhage. Neurosurg Clin N Am. 2010;21(2):365-379.

13. Toda N, Ayajiki K, Okamura T. Cerebral blood flow regulation by nitric oxide: recent advances. Pharmacol Rev. 2009;61(1):62-97.

14. Hanggi D, Steiger HJ. Nitric oxide in subarachnoid haemorrhage and its therapeutics implications. Acta Neurochir (Wien). 2006;148(6): 605-613.

15. Clark LN, Dittrich HC. Cardiac imaging using Optison. Am J Cardiol. 2000;86(4A):4G-18G.

16. Alkan-Onyuksel H, Demos SM, Lanza GM, et al. Development of inherently echogenic liposomes as an ultrasonic contrast agent. J Pharm Sci. 1996;85(5):486-490.

17. Bokor D. Diagnostic efficacy of SonoVue. Am J Cardiol. 2000;86(4A): 19G-24G.

18. Buchanan KD, Huang S, Kim H, Macdonald RC, McPherson DD. Echogenic liposome compositions for increased retention of ultrasound reflectivity at physiologic temperature. J Pharm Sci. 2008;97(6): 2242-2249.

19. Huang SL, Hamilton AJ, Pozharski E, et al. Physical correlates of the ultrasonic reflectivity of lipid dispersions suitable as diagnostic contrast agents. Ultrasound Med Biol. 2002;28(3):339-348.

20. Bevan PD, Karshafian R, Tickner EG, Burns PN. Quantitative measurement of ultrasound disruption of polymer-shelled microbubbles. Ultrasound Med Biol. 2007;33(11):1777-1786.

21. Hamilton A, Huang SL, Warnick D, et al. Left ventricular thrombus enhancement after intravenous injection of echogenic immunoliposomes: studies in a new experimental model. Circulation. 2002;105(23): 2772-2778.

22. Hamilton AJ, Huang SL, Warnick D, et al. Intravascular ultrasound molecular imaging of atheroma components in vivo. J Am Coll Cardiol. 2004;43(3):453-460

23. Huang S, Hamilton AJ, Tiukinhoy SD, et al. Liposomes as ultrasound imaging contrast agents and as ultrasound-sensitive drug delivery agents. Cell Mol Biol Lett. 2002;7(2):233-235.

24. Tiukinhoy-Laing SD, Huang S, Klegerman M, Holland CK, McPherson DD. Ultrasound-facilitated thrombolysis using tissueplasminogen activator-loaded echogenic liposomes. Thromb Res. 2007;119(6):777-784.

25. Huang SL, Kee PH, Kim H, et al. Nitric oxide-loaded echogenic liposomes for nitric oxide delivery and inhibition of intimal hyperplasia. J Am Coll Cardiol. 2009;54(7):652-659.

26. Britton GL, Kim H, Kee PH, et al. In vivo therapeutic gas delivery for neuroprotection with echogenic liposomes. Circulation. 2010; 122(16):1578-1587.
27. Kopechek JA, Abruzzo TM, Wang B, et al. Ultrasound-mediated release of hydrophilic and lipophilic agents from echogenic liposomes. J Ultrasound Med. 2008;27(11):1597-1606.

28. Arita NO, Cohen MF, Tokuda G, Yamasaki H. Fluorometric detection of nitric oxide with diaminofluoresceins (DAFs): applications and limitations for plant NO research. In: Lamattina L, Polacco JC, editors. Nitric Oxide in Plant Growth, Development and Stress Physiology. Berlin, Germany: Springer; 2007.

29. Heydemann A, Huber JM, Kakkar R, Wheeler MT, McNally EM. Functional nitric oxide synthase mislocalization in cardiomyopathy. J Mol Cell Cardiol. 2004;36(2):213-223.

30. Lee JY, Sagher O, Keep R, Hua Y, Xi G. Comparison of experimental rat models of early brain injury after subarachnoid hemorrhage. Neurosurgery. 2009;65(2):331-343

31. Kopechek JA, Kim H, McPherson DD, Holland CK. Calibration of the 1-MHz Sonitron ultrasound system. Ultrasound Med Biol. 2010; 36(10):1762-1766.

32. Zhao X, Sun G, Zhang J, et al. Hematoma resolution as a target for intracerebral hemorrhage treatment: role for peroxisome proliferatoractivated receptor gamma in microglia/macrophages. Ann Neurol. 2007;61(4):352-362.

33. Huang SL, McPherson DD, Macdonald RC. A method to co-encapsulate gas and drugs in liposomes for ultrasound-controlled drug delivery. Ultrasound Med Biol. 2008;34(8):1272-1280.

34. Kim H, Moody MR, Laing ST, et al. In vivo volumetric intravascular ultrasound visualization of early/inflammatory arterial atheroma using targeted echogenic immunoliposomes. Invest Radiol. 2010;45(10): 685-691.

35. Bader KB, Holland CK. Gauging the likelihood of stable cavitation from ultrasound contrast agents. Phys Med Biol. 2013;58(1):127-144.

36. Smith DA, Porter TM, Martinez J, et al. Destruction thresholds of echogenic liposomes with clinical diagnostic ultrasound. Ultrasound Med Biol. 2007;33(5):797-809.

37. Elmalak O, Lovich MA, Edelman E. Correlation of transarterial transport of various dextrans with their physicochemical properties. Biomaterials. 2000;21(22):2263-2272.

38. Sibley CP, Bauman KF, Firth JA. Permeability of the foetal capillary endothelium of the guinea-pig placenta to haem proteins of various molecular sizes. Cell Tissue Res. 1982;223(1):165-178.

39. Dayton PA, Ferrara KW. Targeted imaging using ultrasound. J Magn Reson Imaging. 2002;16(4):362-377.

40. Sabri M, Ai J, Macdonald RL. Nitric oxide related pathophysiological changes following subarachnoid haemorrhage. Acta Neurochir Suppl. 2011;110 Pt 1:105-109.

41. Sehba FA, Bederson JB. Nitric oxide in early brain injury after subarachnoid hemorrhage. Acta Neurochir Suppl. 2011;110 Pt 1:99-103.
International Journal of Nanomedicine

\section{Publish your work in this journal}

The International Journal of Nanomedicine is an international, peerreviewed journal focusing on the application of nanotechnology in diagnostics, therapeutics, and drug delivery systems throughout the biomedical field. This journal is indexed on PubMed Central,

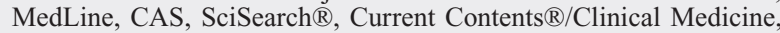

\section{Dovepress}

Journal Citation Reports/Science Edition, EMBase, Scopus and the Elsevier Bibliographic databases. The manuscript management system is completely online and includes a very quick and fair peer-review system, which is all easy to use. Visit http://www.dovepress.com/ testimonials.php to read real quotes from published authors. 